$45 \mid 2010$

Langue française, identité(s) et école(s) : le cas de la minorité catholique au Levant (milieu XIX ${ }^{\mathrm{e}}-\mathrm{XX}^{\mathrm{e}}$

siècles). Représentations identitaires et apprentissage des langues : Europe, bassin méditerranéen ( $\left.\mathrm{XV}\right|^{\mathrm{e}}-\mathrm{XX}{ }^{\mathrm{e}}$ siècles)

\title{
Jocelyne Dakhlia. Lingua franca. Histoire d'une langue en Méditerranée.
}

Actes Sud, 2008, 592 p. ISBN 978-2-7427-8077-8.

\section{Gérard Vigner}

\section{(2) OpenEdition}

Édition électronique

URL : https://journals.openedition.org/dhfles/2485

DOI : $10.4000 /$ dhfles.2485

ISSN : 2221-4038

Éditeur

Société Internationale pour l'Histoire du Français Langue Étrangère ou Seconde

Édition imprimée

Date de publication : 1 juin 2010

Pagination : 210-214

ISSN : 0992-7654

Référence électronique

Gérard Vigner, « Jocelyne Dakhlia. Lingua franca. Histoire d'une langue en Méditerranée. », Documents pour l'histoire du français langue étrangère ou seconde [En ligne], 45 | 2010, mis en ligne le 31 août 2013, consulté le 27 mai 2021. URL : http://journals.openedition.org/dhfles/2485 ; DOI : https://doi.org/ $10.4000 /$ dhfles.2485

Ce document a été généré automatiquement le 27 mai 2021.

(c) SIHFLES 


\section{Jocelyne Dakhlia. Lingua franca. Histoire d'une langue en Méditerranée.}

Actes Sud, 2008, 592 p. ISBN 978-2-7427-8077-8.

\section{Gérard Vigner}

1 Ouvrage qui se donne comme projet de retracer l'histoire d'une langue disparue dont seule subsiste dans les mémoires la dénomination, au sens de langue de contact entre locuteurs de langues différentes et non partagées. Or la lingua franca ou langue franque, dans la version française, a eu une histoire et une géographie. Une histoire qui nous conduit en gros du $\mathrm{XVI}^{\mathrm{e}}$ siècle pour ce qui est au moins de la première description disponible (il s'agit de la description entreprise par un bénédictin, rédempteur de captifs à Alger entre 1578 et 1581 et publiée à Valladolid en 1612), au début des conquêtes coloniales et plus particulièrement à celle de l'Algérie à partir de 1830 (Marcel Cohen dans son ouvrage intitulé Le Parler arabe des juifs d'Alger, publié en 1912, ne put en effet identifier que quelques reliques lexicales de la lingua franca, depuis longtemps disparue des usages, dans le langage du commerce); et une géographie, nous reviendrons plus loin sur cette question, celle de la Méditerranée occidentale. Langue dont les usages sont inséparables de la course barbaresque, des régences ottomanes d'Afrique du Nord et des bagnes qui y sont installés. Tentative méritoire que d'inscrire dans son historicité ce qui fut un pidgin, ou ce que certains appelèrent un «jargon de fortune ", alors même que la plasticité des usages de ce type de langues, l'absence de documents écrits (ce véhiculaire en effet n'a pas engendré de littérature véritable ${ }^{1}$ ), font de la restitution de cette langue commune aux locuteurs variés une tâche particulièrement délicate.

2 Langue que l'on distinguera tout d'abord de la langue en usage dans les royaumes francs d'Orient élaborée dans d'autres conditions par la rencontre de locuteurs venus du royaume capétien, de Normands du sud de l'Italie, d'Occitans et d'Italiens divers. Langue qui donna lieu à une abondante production écrite ${ }^{2} a u$ moins selon les normes de l'époque, et qui à partir de cette « romania d'outre-mer » donna naissance à une variété particulière de français. Alors que notre lingua franca, ici, est bien cette langue composite en usage dans certains milieux, esclaves chrétiens, renégats, navigateurs, 
commerçants en tous genres, autrement dit de tous les milieux liés à la course barbaresque. Langue qui connaitra d'autres dénominations, on parlera encore de franco et de petit mauresque. On notera d'ailleurs la publication par la Chambre de commerce à Marseille, en 1830, d'un dictionnaire destiné notamment à aider les militaires à se faire comprendre de la population, Dictionnaire de la Langue ou Petit Mauresque, suivi de quelques dialogues familiers et d'un vocabulaire de mots arabes les plus usuels; à l'usage des Français en Afrique. La conquête de l'Algérie par la France signera la fin de la langue franque et la transition vers les sabirs, c'est-à-dire la langue des maîtres utilisée dans une version simplifiée.

3 La lingua franca est un mixte de langues à base romane - l'italien y occupe une place importante - dans laquelle on ne peut relever qu'une très faible présence de traits lexicaux et syntaxiques issus de la langue arabe, alors que l'on sait que les locuteurs de langue arabe en firent aussi un important usage. Romanité qui ne peut qu'intriguer alors que la lingua franca est une langue en contexte symétrique dans laquelle l'arabe, ou le turc - n'oublions pas que nous sommes dans le domaine des régences ottomanes aurait pu tout aussi bien trouver sa place. Importance aussi des apports ibériques qui peut être interprétée par l'importance des affrontements entre Espagnols et Ottomans à Tunis, par le siège d'Alger en 1541 par Charles Quint et l'existence de "presides " dans l'ouest du Maghreb. Conflits qui donneront lieu à la réduction en captivité de plusieurs milliers d'hommes, de part et d'autre, et à cette nécessité de pouvoir communiquer, se comprendre et à cette fin d'élaborer une langue qui soit à la portée de tous. Mais la Méditerranée occidentale est d'abord une zone d'affrontement nettement affirmée.

4 Jocelyne Dakhlia va en effet fonder son analyse sur une série de témoignages de voyageurs qui rapporteront dans leurs récits ou descriptions des échantillons de langue qui seront donc toujours des échantillons rapportés dans des formes de transactions qui ne portent que sur des enjeux des plus ordinaires. Nul échange traitant de sujets élaborés et complexes, nul sujet apparemment qui implique véritablement les locuteurs, alors que l'on sait que cette langue fut la langue de contact entre maîtres et esclaves dans le quotidien de la vie de la régence d'Alger par exemple et même en usage chez les diplomates. Véhiculaire au corpus étroit qui pour ces raisons-là n'offre de luimême qu'une représentation tronquée. Dans tous les cas, on observera que ce véhiculaire, à la différence de nombreux autres dans le monde, n'a pas donné lieu à l'émergence d'un créole, c'est-à-dire d'une langue que vont s'approprier certaines populations pour en faire leur langue maternelle. No man's langue, pour reprendre une expression dont Jocelyne Dakhlia fait usage dans un autre ouvrage ${ }^{3}$.

5 Dans une succession de chapitres qui intègrent de nombreuses citations d'époque, l'auteur va progressivement cerner, puis préciser la nature de cette langue dans les liens qui l'inscrivent dans le jeu des autres langues, dans l'identification, autant que faire se peut, des locuteurs. Plusieurs chapitres, très fortement documentés, explorent la géographie de cette langue, aussi bien en terre d'Islam qu'en Europe occidentale. Jocelyne Dakhlia, au rebours de certains témoignages, montre bien le caractère relativement restreint des zones d'usage, usages plus ponctuels notamment quand on s'écarte des rives de la Méditerranée, mais attestés cependant à partir des nombreux témoignages de voyageurs. Souvenons-nous que Jean-Jacques Rousseau lui-même, dans son livre IV des Confessions affirme connaitre cette langue et rend compte de scènes d'échange dans laquelle cette langue est en usage. Ce parcours, attentif, permet en 
même temps de développer toute une réflexion sur la perception des langues durant toutes les périodes considérées et la difficulté, pour les témoins de l'époque, de parvenir à approcher la singularité de cette langue qui, aux yeux de nombreux d'entre eux, passa pendant longtemps pour de l'italien ou de l'espagnol « corrompu ». À quelles conditions une langue composite peut-elle devenir une langue appréhendée dans sa singularité ? Et la succession des témoignages et des analyses montre bien la difficulté des contemporains à percevoir cette langue comme une langue séparée. La polémique se poursuivra jusqu'à la fin du XIX siècle. Lucien Bonaparte devra encore longuement plaider (1877) pour que la langue franque ne soit pas perçue comme une langue déformée par la pratique de locuteurs étrangers. Mais on aurait aimé quelques éclairages plus développés sur cette élaboration d'une langue singulière, séparée, à partir de matériaux pris à d'autres langues en usage dans les mêmes territoires.

Le chapitre consacré à la disparition de la langue franque au moment de la conquête coloniale permet à l'auteur de développer d'utiles réflexions autour de la notion de sabir et de langues en miroir tels le cagayous ou la patahouète qui circulèrent dans l'Afrique du Nord coloniale dans un jeu complexe de relations entre populations aux statuts divers. Tout ceci dans un contexte dans lequel le français, langue "pure", occupe désormais une place prédominante et fausse d'une certaine manière le jeu spontané des locuteurs lorsqu'ils doivent communiquer dans un environnement linguistiquement ouvert.

7 Cette langue de l'entre-deux entre sociétés adverses que nulle communauté en fait ne s'est appropriée pour en faire sa langue, ne peut manquer de susciter une réflexion autour du concept si largement sollicité aujourd'hui de métissage. «Objet métis » en effet, mais qui ne débouche sur aucune fusion, aucune mixité. Langue que l'on ne pourrait investir d'une valeur de rapprochement interculturel, langue d'une certaine proximité, d'un continuum en même temps que de marquage de la différence. Un fait historique, non une valeur en soi. Singularité du destin d'une langue oubliée qui rend compte de configurations originales à un moment où les langues officielles, dans ces mêmes régions, avec la construction des politiques nationales, tentent d'affirmer leurs oppositions réciproques par la valorisation d'un monolinguisme qui se veut toujours exclusif.

8 Ouvrage rédigé dans une langue accessible au non-spécialiste, pourvu d'un abondant appareil de notes, ainsi que d'un index des noms propres, et qui trouve là tout à la fois son intérêt et ses limites. Son intérêt parce qu'il permet de cerner un objet aux contours incertains, dans une relation aux locuteurs peu aisée à déterminer en dehors des témoignages de voyageurs et qui, en l'absence de documents écrits originaux, réclame de la part du chercheur un important travail d'analyse et de contextualisation. Ses limites dans la mesure où un certain nombre de questions théoriques ne sont pas ici abordées. À quelles conditions un véhiculaire peut-il émerger, dès lors qu'existent des langues si l'on peut s'exprimer ainsi, de plein exercice, comme l'arabe, l'hébreu, le français, l'italien, l'espagnol et qui dans certains cas peuvent court-circuiter le stade du véhiculaire ? Ainsi de la Méditerranée orientale qui, en dépit de l'extraordinaire variété des langues en contact, n'a pas donné lieu à l'émergence d'un véhiculaire tel que la lingua franca dans la Méditerranée du Ponant? Faut-il concevoir que les locuteurs de langues romanes dans la Méditerranée du Levant (croisés et négociants en tous genres) disposaient d'une meilleure connaissance de l'arabe, du grec, du turc? Et faut-il supposer alors que les locuteurs en contact dans l'espace méditerranéen occidental 
étaient dépourvus de cette connaissance ou qu'il s'agissait là d'une autre famille de locuteurs, moins lettrés, et recourant plus spontanément à des formes originales pidginisées? Questions qui méritent examen, d'autant qu'il conviendrait de mieux connaitre les usages, en milieux lettrés, de langues telles que l'arabe, les langues romanes ou l'hébreu, qui pouvaient entrer en concurrence avec ceux de la lingua franca. Un certain nombre d'échantillons de cette langue, tels que les rapportent les voyageurs, permettent de se faire une idée des singularités de ce véhiculaire, mais nulle analyse linguistique ici qui permette de définir, non pas une lingua franca pure, mais des modes de dérivation lexicale, un appareillage morphologique propre, qui la différencient d'un italien dégrammaticalisé. Ce qui pose aussi la question des modes d'élaboration de ce type de langue, par alternance codique, par mélange des codes ou mise en relation de différents sous-systèmes. Questions fort complexes qui éloignent d'ailleurs du propos de l'ouvrage et que l'auteur à l'évidence ne voulait pas aborder ici. Histoire d'une autre Méditerranée, histoire d'un " pidgin », une de ces langues sans-papiers que les hommes bricolent à des fins de commodités d'usage et qui méritent certainement d'être mieux connues tant leur présence est universellement attestée.

\section{NOTES}

1. On doit cependant signaler ce texte assez singulier qu'est la Zerbitana, bref poème rédigé dans le courant du XIII ${ }^{\text {e }}$ siècle et qui rend compte d'une dispute entre une Jerbienne, habitante de l'île de Jerba, et un soldat sicilien qui tente de forcer sa porte. En revanche, on ne peut ignorer l'usage que fit Molière de cette langue dans la fameuse scène du Mamamouchi figurant dans Le Bourgeois gentilhomme, strophes inspirées des témoignages rapportés par le chevalier d'Arvieux, diplomate français qui fit de nombreux voyages et missions dans la Méditerranée musulmane et qui fut en quelque sorte l'«expert » auquel s'adressèrent Lully et Molière pour composer leur fameuse scène.

2. On trouvera des éléments de description de cette langue dans l'article de Pierre Nobel, «Écrire dans le Royaume franc : la scripta de deux manuscrits copiés à Acre au XIII siècle ", in Variations linguistiques, koinè, dialectes, français régionaux, Presses universitaires de Franche-Comté, 2003.

3. Jocelyne Dakhlia (dir.), Trames de langues. Usages et métissages linguistiques dans l'histoire du Maghreb, Maisonneuve et Larose, 2004. 